\title{
BMJ Open Quality Electronic alerts for acute kidney injury across primary and secondary care
}

\author{
Joseph Barker (1D , ${ }^{1,2}$ Karl Smith-Byrne, ${ }^{3}$ Oliver Sayers, ${ }^{4}$ Krishan Joseph, ${ }^{5}$ \\ Mark Sleeman, ${ }^{6}$ Daniel Lasserson, ${ }^{7}$ Emma Vaux ${ }^{8}$
}

To cite: Barker J, SmithByrne K, Sayers 0 , et al. Electronic alerts for acute kidney injury across primary and secondary care. BMJ Open Quality 2021;10:e000956. doi:10.1136/ bmjoq-2020-000956

- Additional supplemental material is published online only. To view, please visit the journal online (http://dx.doi.org/10. 1136/bmjoq-2020-000956).

Received 21 February 2020 Revised 19 April 2021 Accepted 21 April 2021

\section{Check for updates}

(C) Author(s) (or their employer(s)) 2021. Re-use permitted under CC BY-NC. No commercial re-use. See rights and permissions. Published by BMJ.

For numbered affiliations see end of article.

Correspondence to Dr Joseph Barker; joseph.barker@nhs.net

\section{ABSTRACT}

Problem In 2009 the National Confidential Enquiry into Patient Outcome and Death suggested only $50 \%$ of patients with acute kidney injury (AKI) receive good standards of care. In response National Health Service (NHS) England mandated the use of electronic AKI alerts within secondary care. However, we recognised AKI is not just a secondary care problem, where primary care has a crucial role to play in prevention, early detection and management as well as post-AKI care.

Methods AKI alerts were implemented in primary and secondary care services for a population of 480000 . Comparisons were made in AKI incidence, peak creatinine following AKI and renal recovery in the years before and after using Byar's approximation $(95 \% \mathrm{Cl})$.

Intervention A complex quality improvement initiative was implemented based on the design and integration of an AKI alerting system within laboratory information management systems for primary and secondary care, with an affixed URL for clinicians to access a care bundle of AKI guidelines on safe prescribing, patient advice and early contact with nephrology.

Results The intervention was associated with an $8 \%$ increase in creatinine testing $(n=32563)$. Hospital acquired AKI detection increased by $6 \%$, while community acquired AKI detection increased by $3 \%$ and AKI stage 3 detected in primary care fell by $14 \%$. The intervention overall had no effect on AKI severity but did improve follow-up testing and renal recovery. Importantly hospital AKI 3 recoveries improved by $22 \%$. In a small number of AKI cases, the algorithm did not produce an alert resulting in a reduction in follow-up testing compared with preintervention levels. Conclusion The introduction of AKI alerts in primary and secondary care, in conjunction with access to an AKI care bundle, was associated with higher rates of repeat blood sampling, AKI detection and renal recovery. Validating accuracy of alerts is required to avoid patient harm.

\section{INTRODUCTION}

\section{Problem description}

Acute kidney injury (AKI) is a common, costly and often preventable disorder associated with significant morbidity and mortality. It is detected in $13 \%-18 \%$ of UK hospital admissions and is becoming more common in primary care. ${ }^{2}$ AKI increases the risk of developing chronic kidney disease (CKD). ${ }^{3}$ A rise in creatinine of $\geq 500 \mu \mathrm{mol} / \mathrm{L}$ is associated with as much as a 6.5-fold increase in mortality during inpatient admissions over 3-7 days. ${ }^{4}$

In 2009 the UK National Confidential Enquiry into Patient Outcome and Death published a review examining all cases of patients who died in National Health Service (NHS) hospitals with a primary diagnosis of AKI. The review included 976 deaths across 216 hospitals and required each case to be reviewed by the patient's managing consultant. It concluded that only $50 \%$ of patients were considered to have received an overall 'good' standard of care, with the majority of care deficit being clinically rather than organisationally mediated. With respect to AKI this indicated a lack of recognition; a lack awareness of the inherent risk of AKI; a poor understanding of the pathophysiology of the condition and/or an inadequate knowledge of its management among clinicians. ${ }^{1}$

\section{Available knowledge}

In response several NHS hospitals undertook quality improvement projects implementing automated AKI electronic alerts (AKI alerts) together with AKI treatment bundles. These interventions pushed AKI alerts to clinicians through their blood results reporting systems to highlight AKI diagnosis and grade severity as well as offer management advice. Nottingham University Hospital in 2011 was the first to implement an AKI alert system in a large NHS hospital, though the impact of this intervention was not evaluated. ${ }^{5}$ In 2012 University College London Hospital implemented the first primary care AKI alert system, though this was only qualitatively assessed with primary care practitioners reporting it was a useful service that altered practice. ${ }^{6}$ In 2013 Aintree University Hospital implemented an alert system within secondary care paired with an AKI treatment bundle and patient information leaflet reporting large quantitative reductions in 30-day mortality, length of stay and progression of AKI severity. ${ }^{7}$ These projects demonstrated the feasibility of implementing AKI alerts pushed to clinicians through the laboratory information 
management systems and in response the 2014 NHS England Patient Safety Alert mandated the adoption of AKI alert systems via the introduction of a Commissioning for Quality and Innovation target (CQUIN) in secondary care with adoption in primary care to be considered at a later date. ${ }^{8-10}$ Subsequent publications have generated mixed results on the efficacy of AKI alerts on patient outcomes. ${ }^{11-14}$

\section{Rationale}

In recognising primary care has a crucial role to play in the prevention, early detection and management of AKI, as well as post-AKI care, a yearlong quality improvement programme to standardise AKI care pathway across the trust to include primary care was undertaken prior to the adoption of the CQUIN, described in detail by Vaux. ${ }^{15}$ As a result of this, and an extensive cross boundary clinician engagement programme, it was felt the system was ready for implementation of alerts across both secondary and primary care. This context facilitated an evaluation of the impact of an AKI alert system across an entire health system inclusive of both primary and secondary levels of care described in this paper.

\section{Specific aim}

To reduce AKI severity and improve renal recovery through earlier recognition of AKI, safer prescribing, earlier contact with nephrology, improved patient follow-up, better provision of patient information and where possible community led management of AKI.

\section{METHODS \\ Context}

Four Clinical Commissioning Groups (CCGs) representing the NHS bodies responsible for commissioning care in their region. These included North and West Reading CCG, Newbury and District CCG, South Reading CCG, Wokingham CCG totalling an area covering 54 general practices and Royal Berkshire Hospital serving a total population of approximately 480000 patients.

\section{Intervention}

In the year prior to the implementation of AKI alerts, an innovative intervention used the combination of three approaches: information design, human factors and quality improvement methodology in the collaborative design and development of AKI care bundles (online supplemental appendices 1 and 2) to support complex clinical decision-making. ${ }^{15}$ Iterative user and stakeholder consultation across primary and secondary care (including junior doctors, general practitioners (GPs), GP out of hours services, hospital and community pharmacists, consultants, nurses, patients, dieticians, information design consultants, human factors specialist) was employed to develop a design response that was sensitive to user experience and need, culminating in simulation testing of a near final prototype. The development of supplementary awareness-raising materials, a communications and knowledge transfer package built around 'tAKIng care, tAKIng note', to promote understanding of the usefulness of the intervention (in terms of benefit to both staff and patients), so ensuring its sustainability, alongside face-to-face stakeholder implementation events using worked through examples helped communicate and support care bundle implementation. The multi-professional approach using semi-structured focus groups, case-based discussion, supported by project management support, was transformational in developing effective engagement, collaboration and data sharing between health professionals within and across the local and regional health economy. Easy to access care bundles was critical to their use and coimplementation of the new approach across primary and secondary care and involved both clinicians and laboratory staff. The AKI alerts were then implemented for both primary and secondary care in June 2015 with AKI incidence data collected the year prior to intervention and the year following intervention. All learning was reinforced through our 2015/2016 AKI CQUIN focused on improving quality of information in the discharge letter of a patient who had had an episode of AKI during a hospital admission. ${ }^{916}$ This identified that educational and awareness initiatives alone were not sufficient in changing practice and mandating user review of AKI aftercare information for GPs in patients with AKI alerts was necessary for a sustained solution.

\section{Study of the intervention}

Anonymised creatinine results were collected for all patients over 18 years of age having blood tests performed by the Royal Berkshire Hospital biochemistry laboratory between July 2014 and October 2016 along with location data from which the blood test was requested and sampled. This laboratory was the routine service used for processing blood results for all 54 primary care practices included in the study as well as secondary care facilities. No changes were made to NHS standard of care for blood taking, transportation, storage or processing of samples.

AKI annual incidence was determined independent of the alert system embedded in the laboratory information management systems using the 2014 NHS England AKI Algorithm Patient Safety Alert. ${ }^{17}$ Renal function data coming from nephrology departments were excluded from the analysis. AKI incidence rates per 100000 population were calculated using Byar's approximation with CIs of $95 \%$ (95\% CI). Peak creatinine was determined as the highest creatinine within 30 days of AKI (95\% CI). Recovery to baseline renal function was determined as creatinine values falling within $10 \%$ of pre-AKI creatinine reached within 90 days (95\% CI). Results were stratified by location the blood was sampled and requested from; these included community hospital inpatient setting='Community inpatient'; community outpatient setting; ='Community outpatient'; community emergency setting including emergency department, acute medical unit and clinical decision units='ED/AMU/CDU'; hospital inpatient settings='Hospital inpatient'; primary care $=$ 'General 
Table 1 Demographic characteristics of participants and number of creatinine tests by location and phase of study

\begin{tabular}{lll}
\hline Variables & Year 1 & Year 2 \\
\hline Population* & 478168 & 481381 \\
\hline $\begin{array}{l}\text { Unique } \\
\text { individuals } †\end{array}$ & 155127 & 164777 \\
\hline Age & $61.7(61.7$ to 61.8) & 60.5 (60.5 to 60.6) \\
\hline Male & $54.8 \%$ & $54.8 \%$ \\
\hline Female & $45.2 \%$ & $45.2 \%$ \\
\hline Creatinine testing location & \\
\hline Community inpatient & 4239 & 4807 \\
\hline Community outpatient & 863 & 768 \\
\hline ED/AMU/CDU & 47200 & 53769 \\
\hline General practice & 203580 & 224103 \\
\hline Hospital inpatient & 80755 & 82647 \\
\hline Hospital outpatient & 60964 & 64046 \\
\hline $\begin{array}{l}\text { Combined community } \\
\text { locales }\end{array}$ & 312607 & 342686 \\
\hline Combined inpatient & 84994 & 87454 \\
locales & & 430140 \\
\hline Total & 397601 & \\
\hline
\end{tabular}

Age $=$ mean $(95 \% \mathrm{Cl})$.

*Population derived from Office for National Statistics Clinical Commissioning Group population estimates. ${ }^{20}$

†Number of unique individuals across both study years: 228903 . ED/AMU/CDU, emergency department, acute medical unit and clinical decision units.

Practice'; and finally hospital outpatient setting='Hospital outpatient'. These locales were combined to create community acquired and hospital acquired AKI categories; with community acquired AKI locations including community outpatients, community emergency settings, primary care and hospital outpatients. Hospital acquired AKI refers to inpatient development of AKI in either community or hospital inpatients.

\section{RESULTS}

In the year following intervention the total number of unique individuals undergoing renal function testing increased by $9650(+6 \%)$, while total testing increased by 32539 tests $(+8 \%)$. Stratifying results by location shows an increase in renal function testing in most locations, with the majority of this increase in primary care $+10 \%(20523$ tests) compared with inpatient testing of only $+3 \%$ (2460) (see table 1).

The implementation of the AKI alerts was associated with greater AKI detection of $+5 \%$ (2500-2613 per 100000 population) which when stratified by location shows an increase in both hospital acquired AKI of +6\% (14321516 per 100000 population) and community acquired AKI of $+3 \%$ (1068-1096 per 100000 population). Notably AKI stage 3 detection decreased in primary care by $-14 \%$ (31-26 per 100000 population) with a concomitant increase in hospital inpatient AKI 3 detection of $+10 \%$
(202-222 per 100000 population) (table 2). Aggregated results showed an increase in AKI 1 detection of $+0.02 \%$ (1648-1690 per 100000 population), an increase in AKI 2 detection of $+7.4 \%$ (459-493 per 100000 population) and an increase in AKI 3 detection by 9\% (393-429 per 100000 population) (see table 2 ).

The effect of the implementation on AKI alerts on peak creatinine during AKI was to remain static in all severities of AKI in all stratified locations with the exception of hospital inpatient AKI stage 3 which increased from $459 \mathrm{\mu mol} / \mathrm{L}(440-477)$ to $535 \mathrm{\mu mol} / \mathrm{L}$ (508-563). Aggregated results show an increase in AKI stage 2 from 243 $\mu \mathrm{mol} / \mathrm{L}(234-252)$ to $262 \mu \mathrm{mol} / \mathrm{L}(254-270)$ and AKI stage 3 from $506 \mu \mathrm{mol} / \mathrm{L}(489-523)$ to $548 \mu \mathrm{mol} / \mathrm{L}$ (527569 ) following the implementation of AKI alerts (online supplemental table A).

Follow-up renal function testing within 7 days increased overall by $+5 \%$ ( $76-80$ per 100 episodes of AKI) after AKI (table 3). Stratification in all locations and in all severity categories of AKI mirrored improvement in follow-up tests with the most significant improvement in follow-up in community outpatient $+140 \%$ (37-80 per 100 episodes of AKI), GP by $+38 \%$ (37-51 per 100 episodes of AKI) and hospital outpatient by $+16 \%$ (49-57 per 100 episodes of AKI). Overall community acquired AKI follow-up seemed to benefit more from the implementation of AKI alerts than hospital acquired AKI with community AKI follow-up improving $+11 \%$ (63-70 per 100 episodes of AKI) compared with $+1 \%$ (86-87 per 100 episodes of AKI) (see table 3).

Renal recovery to within $10 \%$ of baseline renal function improved across all severities of AKI, while follow-up tests to 90 days were not affected (online supplemental table B). Stratification by location showed most clinically significant improvement in recovery in hospital inpatients AKI stage 3, improving by 7.7 cases per 100 AKI cases (22\% improvement). Community acquired AKI recovery improved by 4 cases per 100 (9\% improvement) and hospital acquired AKI recovery improved by 3.6 cases per 100 cases of AKI (7\% improvement) (see figure 1 and online supplemental table B).

Post hoc analysis of renal function data yielded a $0.55 \%$ inaccuracy of alerts pushed to clinicians in the study due to algorithm failure, including $0.17 \%$ (724 per 430 000 tests) of tests failing to raise an alert for AKI, $0.02 \%$ (102 per 430000 tests) of tests underestimating an AKI and $0.36 \%$ (1553 per 430000 tests) overestimating AKI severity. This provided the opportunity to compare the 724 patients with AKI that were not pushed alerts to those that were in table 3. Overall follow-up creatinine testing within 7 days were similar between the groups however stratification by location shows differences in community acquired AKI follow-up with a reduction of $6.6 \%$. Stratification by AKI severity within the groups showed no real difference in AKI 1 follow-up, with 487 out of 609 patients followed up (80\%), however, AKI 2 and AKI 3 showed reductions in follow-up of $25 \%$ (only 13 of 33 followed up) and $47 \%$ (34 of 82 followed up), respectively (table 3 ). 
Table 2 Number of cases of AKI per 100000 patients (95\% Cl) by location of creatinine testing and phase of study

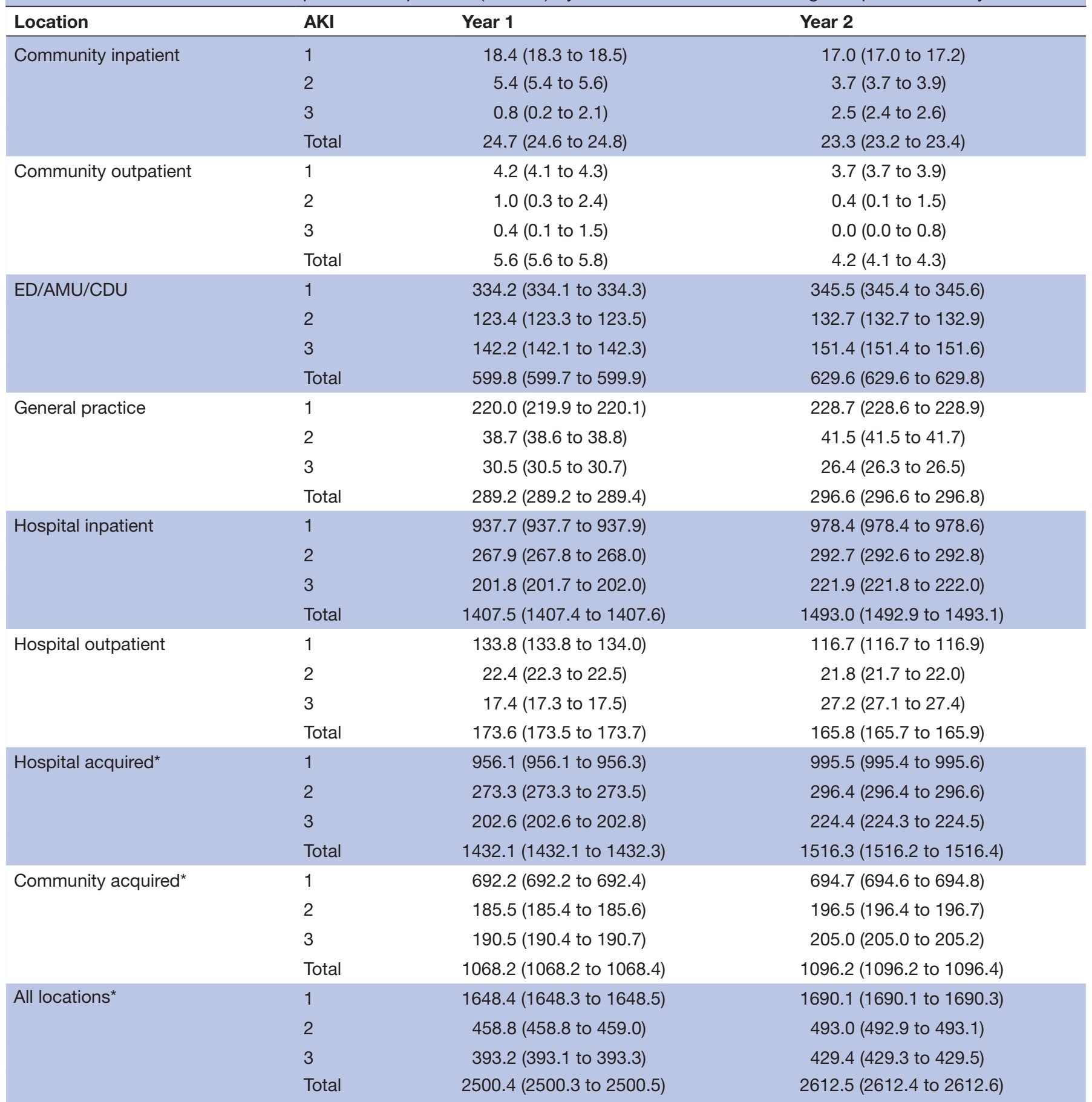

${ }^{\star}$ Aggregated location results.

AKI, acute kidney injury; ED/AMU/CDU, emergency department, acute medical unit and clinical decision units.

\section{DISCUSSION}

\section{Summary}

Our results demonstrate that the introduction of an AKI alert system in conjunction with an AKI care bundle across both primary and secondary care, delivered in the context complex quality improvement initiative, resulted in significant increases in creatinine testing and AKI detection, and importantly improved follow-up and renal recovery following AKI.
The system had to be ready for change. Critical to the introduction of the AKI alert system was the time taken in the preceding year to understand AKI care pathways and capture user experience of care bundles across primary and secondary care. The foundations for this collaborative work had been built though the then Strategic Clinical Networks, cross boundary working to implement the 2014 National Institute of Clinical Excellence CKD guidelines and quality improvement work with primary care on 
Table 3 Patients follow-up with blood tests within 7 days per 100 episodes of AKI by AKI severity and location (95\% CI)

\begin{tabular}{llll}
\hline Variable & Year $\mathbf{1}$ follow-up & Year 2 follow-up & Year 2 follow-up in patients without alert \\
\hline AKI 1 & $73.8(73.8$ to 73.8$)$ & $78.2(78.2$ to 78.2$)$ & $80.0(76.6$ to 83.0$)$ \\
\hline AKI 2 & $81.1(81.1$ to 81.2$)$ & $84.5(84.4$ to 84.5$)$ & $61.0(43.7$ to 75.3$)$ \\
\hline AKI 3 & $78.4(78.4$ to 78.5$)$ & $80.1(80.1$ to 80.2$)$ & $41.5(31.4$ to 52.3$)$ \\
\hline Community inpatient & $64.4(64.1$ to 65.1$)$ & $66.1(65.7$ to 66.8$)$ & $61.1(38.6$ to 79.7$)$ \\
\hline Community outpatient & $37.0(35.7$ to 39.6$)$ & $80.0(78.1$ to 83.6$)$ & $50.0(9.5$ to 90.5$)$ \\
\hline ED/AMU/CDU & $79.6(79.6$ to 79.7$)$ & $81.9(81.9$ to 82.0$)$ & $73.6(65.4$ to 80.5$)$ \\
\hline General practice & $37.1(37.1$ to 37.2$)$ & $50.9(50.9$ to 51.0$)$ & $27.4(18.5$ to 38.6$)$ \\
\hline Hospital inpatient & $85.9(85.9$ to 86.0$)$ & $87.2(87.1$ to 87.2$)$ & $89.9(86.7$ to 92.4) \\
\hline Hospital outpatient & $48.8(48.7$ to 48.9$)$ & $57.3(57.2$ to 57.4$)$ & $55.2(43.4$ to 66.5$)$ \\
\hline Hospital acquired* & $85.6(85.5$ to 85.6$)$ & $86.8(86.8$ to 86.9$)$ & $88.7(85.5$ to 91.3$)$ \\
\hline Community acquired ${ }^{*}$ & $63.1(63.1$ to 63.2$)$ & $69.8(69.8$ to 69.8$)$ & $56.5(50.5$ to 62.2$)$ \\
\hline All AKI* & $76.0(75.9$ to 76.0$)$ & $79.7(79.7$ to 79.7$)$ & $76.7(73.4$ to 79.6$)$ \\
\hline
\end{tabular}

${ }^{*}$ Aggregated location results.

AKI, acute kidney injury; ED/AMUDU, emergency department, acute medical unit and clinical decision units.

prescribing: polypharmacy, inappropriate and/or excessive prescribing. Trusted and engaged relationships had been built across shared visions for change and founded on a proven track record of collaborative improvements. Case based discussion, worked through examples to bring to life use of a care bundle in practice, ease of access to any new tools and recognised value of an intervention were essential groundwork for readiness of stakeholders for the introduction of the AKI alert system.

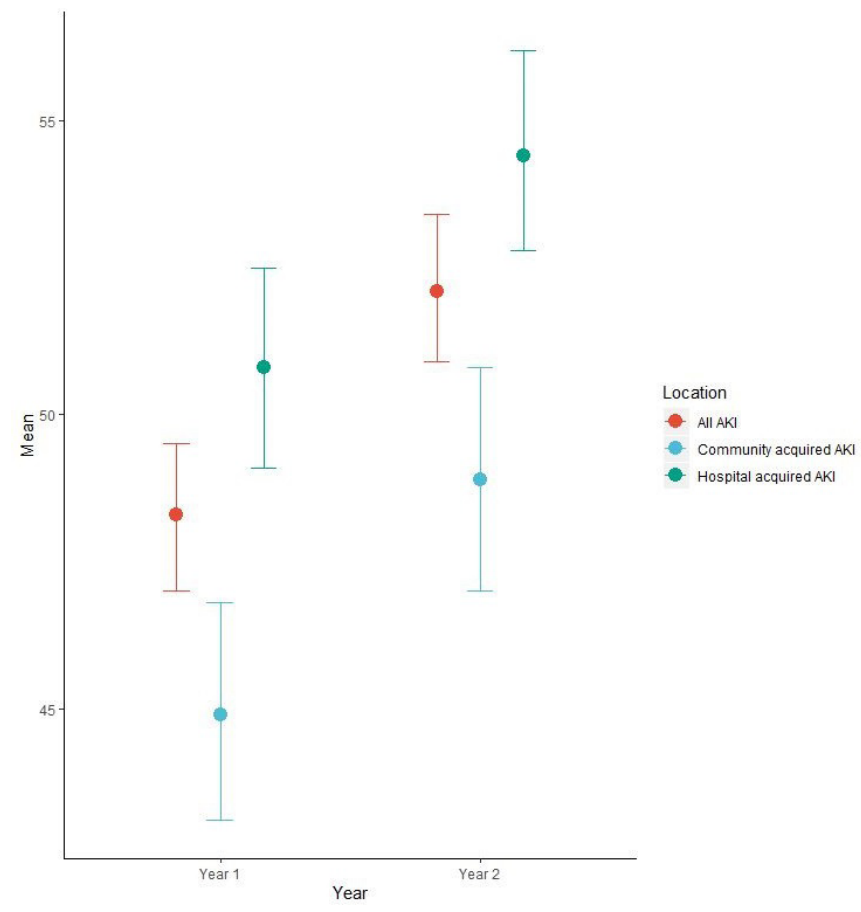

Figure 1 Summary data for acute kidney injury (AKI) recovery to within $10 \%$ of baseline at 90 days per 100 cases of $\mathrm{AKI}$ wherebloods available within 90 days per by $\mathrm{AKI}$ location.
The AKI alert incidence described in our study population is higher than described by Holmes et al in a Welsh population and Aiyegbusi et al in a Scottish population: 2613 versus 2065 and 2362 AKI alerts per 100000 patients, respectively. ${ }^{14} 18$ The overall association of increased creatinine testing and increased AKI detection following the implementation of electronic alerts for AKI is consistent with the findings of Aiyegbusi $e t a l$, who similarly compared annual AKI incidence data from their laboratory information management system preimplementation and post implementation of AKI alerts across primary and secondary care in NHS Tayside, Scotland. The magnitude of increase in incidence is however grossly different, $+17 \%$ versus our $5 \%$. This could be explained by the codesign process of our intervention occurring within the pre-implementation comparator cohort data collection period, serving to raise practitioner salience to AKI independent of AKI alerts and thus to reduce our estimation in the effect of the AKI alert intervention alone. The context in which our intervention took place, with a focus on community led management of AKI within the care bundle, may also explain the gross differences in AKI incidence detected in primary care following the implementation, with Aiyegbusi et al detecting a $60 \%$ reduction in primary care AKI detection and concomitant 13\% increase in AKI admissions, compared with our more stable AKI incidences in both primary care and locations within the acute admissions pathway. This serves to highlight that laboratory information management system alerts alone can inadvertently redirect patient flows and interventions, as with ours, should be designed and implemented to account for this or services may require urgent redesign as a result. Our data also agree with the direction of improvement in AKI follow-up described by Aiyegbusi et al but with similar discrepancy in magnitude as outlined previously. ${ }^{14}$ 
This study is the first of its kind to describe the effect of an AKI alert on the primary outcome of renal recovery. The vast majority of the literature uses end points such mortality, length of stay and requirement for renal replacement therapy which are less likely to capture the more subtle effects of an AKI alert system, particularly in management of less severe AKI. ${ }^{19}$ Stratification by location showed most clinically significant improvement in recovery in hospital inpatients AKI stage 3 but with similar rates of improvement in renal recovery for community and hospital acquired AKIs at all stages.

Finally, our study identified inaccurate AKI alerts in $<1 \%$ of cases, similar to Aiyegbusi et al. ${ }^{14}$ This provided opportunity to compare clinician follow-up behaviour in cases by whether an alert was provided. These data suggest more severe AKI was less likely to be followed up in the cohort without alerts, and concerningly 7-day follow-up dropped from pre-implementation levels. This suggests that the implementation of inaccurate alerts may cause patient harm and any implementation of an electronic AKI alert system must undergo a strict quality control process to avoid adverse events.

\section{Interpretation}

The implementation of an AKI alert system embedded into the laboratory information management system within primary care yielded similar results to secondary care in the improved detection, follow-up and recovery from AKI. Implementation of AKI alerts should take place in the context of meaningful key stake-holder involvement and clarity in how to respond to alerts. We advocate for strict quality control process to validate the accuracy of alert algorithms prior to implementation to reduce the risk of harm to patients from inaccurate alerts.

\section{Limitations}

It is important to highlight the intervention evaluated was a complex package of the introduction of AKI alerts linked to an AKI care bundle supported by supplementary awareness-raising materials, a communications and knowledge transfer package and therefore external validity of the results may be limited by the unique nature of this complex intervention. The intervention effects on long-term health morbidity and mortality were not assessed, instead renal recovery has been used as a surrogate marker which is known to be associated with the development of CKD, cardiovascular disease, the need for chronic dialysis and mortality over time. ${ }^{19}$ This intervention was conducted in one hospital and its surrounding primary care centres, and the results may not generalise to other settings. The static post-hoc data analysis is a possible source of error where data may not align with the dynamic output of a live algorithm running on the data as it stood at that point in time. The comparison of data to the cohort for which no AKI alerts were received were non-randomised and therefore conclusions drawn may be biased.

\section{CONCLUSIONS}

This study supports the inclusion of primary care in the use of AKI alerts. We recommend that the accuracy of AKI alert algorithms is validated prior to implementation to ensure appropriate management for, and follow-up of patients after AKI.

Author affiliations

${ }^{1}$ Oxford University Clinical Academic Graduate School, NIHR Oxford Biomedical

Research Centre, Oxford, UK

${ }^{2}$ Department of Cardiovascular Sciences, University of Leicester, Leicester, UK ${ }^{3}$ Genomic Epidemiology Branch, Section of Genetics, International Agency for

Research on Cancer, Lyon, France

${ }^{4}$ Independent Scholar, London, UK

${ }^{5}$ Department of Opthalmology, Oxford University Hospitals NHS Trust, Oxford, UK

${ }^{6}$ Department of Pathology, Royal Berkshire NHS Foundation Trust, Reading, UK

${ }^{7}$ Department of Health Sciences, Warwick Medical School, University of Warwick, Coventry, UK

${ }^{8}$ Department of Nephrology, Royal Berkshire NHS Foundation Trust, Reading, UK

Twitter Emma Vaux @VauxEmma

Contributors EV was primarily responsible for conceiving, planning and implementing the project. MS oversaw data collection and retrieval from the laboratory information management system. DL provided strategic oversight for project evaluation. JB was primarily responsible for data analysis with KS-B and OS. KJ wrote a narrative literature review to inform manuscript write up. JB wrote up the project where all authors provided critical insight for manuscript revisions. JB, $\mathrm{DL}$ and $\mathrm{EV}$ are the overall content guarantors.

Funding JB is supported by National Institute for Health Research (NIHR), undertaken as part of the Oxford UK Academic Foundation Programme and Leicester Academic Clinical Fellowship with no formal grant/award number. DL is supported by the NIHR Applied Research Collaboration (ARC) West Midlands and the NIHR Community Healthcare MedTech and In Vitro Diagnostics Co-operative (MIC) at Oxford Health NHS Foundation Trust.

Disclaimer The views expressed are those of the authors and not necessarily those of the NIHR, the NHS or the Department of Health and Social Care.

Competing interests None declared.

Patient consent for publication Not required.

Ethics approval This study was conducted as a service evaluation and registered as an audit with Royal Berkshire Hospital NHS Foundation Trust. All data extracted from the laboratory information management systems was not identifiable to individual patients. Only clinical staff had access to routinely collected healthcare data and provided anonymised data for analysis by the evaluation team. The intervention was a development of clinical service and not a randomised intervention. In order to plan future investment in AKI care, the Trust management wished to determine if the intervention was associated with an improvement in clinical and process of care measures.

Provenance and peer review Not commissioned; internally peer reviewed.

Supplemental material This content has been supplied by the author(s). It has not been vetted by BMJ Publishing Group Limited (BMJ) and may not have been peer-reviewed. Any opinions or recommendations discussed are solely those of the author(s) and are not endorsed by BMJ. BMJ disclaims all liability and responsibility arising from any reliance placed on the content. Where the content includes any translated material, BMJ does not warrant the accuracy and reliability of the translations (including but not limited to local regulations, clinical guidelines, terminology, drug names and drug dosages), and is not responsible for any error and/or omissions arising from translation and adaptation or otherwise.

Open access This is an open access article distributed in accordance with the Creative Commons Attribution Non Commercial (CC BY-NC 4.0) license, which permits others to distribute, remix, adapt, build upon this work non-commercially, and license their derivative works on different terms, provided the original work is properly cited, appropriate credit is given, any changes made indicated, and the use is non-commercial. See: http://creativecommons.org/licenses/by-nc/4.0/.

ORCID iD

Joseph Barker http://orcid.org/0000-0002-5483-9608 


\section{REFERENCES}

1 National Confidential Enquiry into Patient Outcome and Death. Adding Insult to Injury [Internet].. UK: NCEPOD, 2019: p. 35.. https:// www.ncepod.org.uk/2009report1/Downloads/AKI_report.pdf

2 Acute kidney injury: prevention, detection and management Guidance and guidelines | NICE [Internet]. Nice.org.uk, 2019. Available: https://www.nice.org.uk/guidance/cg169/chapter/ Introduction\#

3 Coca SG, Singanamala S, Parikh CR. Chronic kidney disease after acute kidney injury: a systematic review and meta-analysis. Kidney Int 2012;81:442-8.

4 Chertow GM, Burdick E, Honour M, et al. Acute kidney injury, mortality, length of stay, and costs in hospitalized patients. J Am Soc Nephrol 2005;16:3365-70.

5 Porter CJ, Juurlink I, Bisset LH, et al. A real-time electronic alert to improve detection of acute kidney injury in a large teaching hospital. Nephrology Dialysis Transplantation 2014;29:1888-93.

6 Alavijeh OS, Bansal J, Hadfield K, et al. Implementation of an automated primary care acute kidney injury warning system: a quantitative and qualitative review of 2 years of experience. Nephron 2017;135:189-95.

7 Chandrasekar T, Sharma A, Chamberlain P, et al. TO023STOPAKIPROJECT: a streamlined approach to AKI management leads to reduction OFIN-HOSPITAL mortality, length of stay and AKI progression. Nephrology Dialysis Transplantation 2016;31:i70-1.

8 NHS England. Standardising the early identification of Acute Kidney Injury [Internet]. London: Renal Association UK Renal Registry, 2014: 1. https://www.england.nhs.uk/patientsafety/wp-content/ uploads/sites/32/2014/06/psa-aki2.pdf

9 NHS England Contracting and Incentives Team Commissioning Strategy. Annex A: commissioning for quality and innovation (CQUIN) 2015/16 guidance templates for use with the NHS standard contract 2015/16]. London: NHS England, 2015: 1-52. https://www.england. nhs.uk/statistics/wp-content/uploads/sites/2/2014/07/CQUINGuidance-2015-16-PDF-641KB.pdf

10 Thinkkidneys.nhs.uk. Acute kidney injury best practice guidance: responding to $\mathrm{AKI}$ warning stage test results for adults in primary care, 2021. Available: <https://www.thinkkidneys.nhs.uk/aki/wpcontent/uploads/sites/2/2016/10/RespondingtoAKI-Warning-StageTest-Results-for-Adults-in-Primary-Care.pdf> [Accessed 18 Mar 2021].

11 Khandaker M, Taha L, Fairclough C, et al. SO041INCIDENCE and mortality of patients with acute kidney injury in hospitalised patients using the NHS England AKI algorithm. Nephrology Dialysis Transplantation 2016;31:i18-19.

12 Lachance P, Villeneuve P-M, Rewa OG, et al. Association between e-alert implementation for detection of acute kidney injury and outcomes: a systematic review. Nephrol Dial Transplant 2017;32:gfw424.

13 West Midlands Acute Medicine Collaborative. The impact of the NHS electronic-alert system on the recognition and management of acute kidney injury in acute medicine. Clin Med 2019;19:109-13.

14 Aiyegbusi O, Witham MD, Lim M, et al. Impact of introducing electronic acute kidney injury alerts in primary care. Clin Kidney $J$ 2019;12:253-7.

15 Vaux E. Designing care bundle documentation to support the recognition and treatment of acute kidney injury: a route to quality improvement, in design for health. In: Tsekleves E, Cooper R, eds. Design for health. Taylor \& Francis Ltd, 2017: 170-90.

16 Reschen ME, Vaux E. Improving the completeness of acute kidney injury follow-up information in hospital electronic discharge letters. BMJ Open Qual 2017;6:e000022.

17 NHS England. Algorithm for detecting Acute Repeat Kidney Injury (AKI) based on serum creatinine changes with time [Internet]. London: Renal Association, UK Renal Registry, 2014: 1. https://www. england.nhs.uk/wp-content/uploads/2014/06/psa-aki-alg.pdf

18 Holmes J, Rainer T, Geen J, et al. Acute kidney injury in the era of the AKI E-Alert. Clin J Am Soc Nephrol 2016;11:2123-31.

19 Forni LG, Darmon M, Ostermann M, et al. Renal recovery after acute kidney injury. Intensive Care Med 2017;43:855-66.

20 Ons.gov.uk. Clinical commissioning group population estimates (National Statistics) - Office for National Statistics, 2019. Available: https://www.ons.gov.uk/peoplepopulationandcommunity/populati onandmigration/populationestimates/datasets/clinicalcommissionin ggroupmidyearpopulationestimates [Accessed 23 Nov 2019]. 